\title{
RUNX3 loss turns on the dark side of TGF-beta signaling
}

\section{Vaidehi Krishnan and Yoshiaki Ito}

The cytokine TGF- $\beta$ is well-known to play the "Jekyll and Hyde" with cancer cells [1]. On one hand, TGF- $\beta$ signaling prevents carcinogenesis in earlystage certain cancers by mediating cell cycle-inhibition and apoptosis. On the other hand, TGF- $\beta$ promotes carcinogenesis in late-stage cancers by inducing invasion, migration and metastasis, partly by the induction of epithelial-to-mesenchymal transition (EMT). Understanding the factors that determine whether TGF- $\beta$ engages in tumor suppression or tumor promotion has remained a subject of intrigue and clinical interest.

In this regard, earlier studies have shown that the RUNX family of proteins influence TGF- $\beta$ signaling through multiple mechanisms. The RUNX genes, RUNX1 and $R U N X 3$ in particular, are frequently inactivated in human cancers at different stages of carcinogenesis [2]. The RUNX proteins are multifunctional and protect cells from transformation by regulating WNT, Ras-ERK, YAP, BMP, Notch, Mitosis, DNA repair and TGF- $\beta$ in a contextual manner [2]. Mechanistically, RUNX proteins control these diverse tumor-suppressive networks either by transcriptional regulation via canonical DNA-binding or by non-transcriptional mechanisms.

Historically, the co-operation between the TGF- $\beta$ signaling and RUNX proteins was discovered during the study of immunoglobulin (IgA) transcription in B lymphocytes. RUNX proteins were shown to physically interact with SMADs, the molecular workhorses of the TGF- $\beta$ pathway to regulate immunoglobulin transcription. Along similar lines, RUNX proteins together with the SMADs regulate TGF- $\beta$-dependent transcription of the cycle inhibitor, p21, and the apoptosis inducer, Bim. Hence, epithelial cells derived from RUNX3-deficient mice were impaired for $\mathrm{p} 21$ and Bim expression and displayed spontaneous EMT [3-5]. In the above-mentioned cellular contexts, RUNX3 deficiency dampens the tumorsuppressive arm of the TGF- $\beta$ signaling pathway.

In our recent work, we have uncovered that the loss of RUNX3 sways TGF- $\beta$ signaling towards tumor promotion [6]. Utilising a non-small cell lung cancer model of TGF- $\beta$-mediated EMT, we found that the loss of $R U N X 3$ promoted oxidative DNA damage when exposed to exogenous TGF- $\beta$. TGF- $\beta$ is known to stimulate ROS production mainly through elevated SMAD-dependent pro-oxidant NOX4 expression. In our mechanistic studies, RUNX3 counteracted TGF- $\beta$-dependent ROS accumulation by upregulation of a redox regulator, Heme oxygenase 1 (HMOX1 or HO-1). HMOX1 is a metabolic enzyme that catalyzes the production of bilirubin, a potent anti-oxidant. The oxidative-DNA damage that accompanied the loss of $R U N X 3$, in turn, triggered cellular senescence accompanied by the expression of inflammatory cytokine and chemokines, called as the senescence-associated secretory phenotype (SASP). Of note, increased SASP has recently assumed a clinical relevance given its ability to amplify carcinogenesis in a paracrine manner [7]. Consistently, lung adenocarcinomas harbouring concurrent TGF- $\beta$ gene expression signature with $R U N X 3$ loss displayed higher levels of genomic instability and poorer survival. In other words, RUNX3 deficiency augments the tumor-promoting arm of the TGF- $\beta$ signaling pathway by exacerbating DNA damage and genomic instability.

Taken together, our study exemplifies how the TGF- $\beta$ signaling pathway is rendered more tumorigenic upon the loss of RUNX3 (Figure 1). The induction of genomic instability in a cell-extrinsic manner is perhaps another ill-consequence of pro-carcinogenic TGF- $\beta$ signaling. Second, RUNX3 protects genomic integrity through $H M O X 1$ transcriptional regulation although the underlying molecular basis needs future studies. Third, similar to RUNX3, lower RUNX1-induced DNA damage accumulation in the presence of TGF- $\beta$, indicating a conservation of function within this family of transcription factors. Fourth, the DNA double strand breaks generated by loss of $R U N X 3$ triggered cellular senescence upon TGF- $\beta$ exposure in an ATM- and ATR-dependent manner. Thus, TGF- $\beta$-elicited cell fate can be modulated by DNA damage response (DDR) kinases. Lastly, the findings are consistent with our earlier study on the role of RUNX1 and RUNX3 as regulators of DNA repair in a nontranscriptional manner. By facilitating the recruitment of DNA repair protein FANCD2 to sites of damage, RUNX proteins were shown to regulate the Fanconi anemia pathway of DNA repair [8]. It is plausible that the RUNX proteins regulate a larger repertoire of DNA repair processes, emphasising their role as unique tumor suppressors with genome maintenance function.

In conclusion, the complexities underlying TGF- $\beta$ signalling present a challenge; but these complexities can be converted into a therapeutic opportunity. Based on our studies and work from others, RUNX3 constitutes at least one important node that determines whether TGF- $\beta$ operates as Jekyll or Hyde in cancers. Manipulating 
genetic networks downstream of RUNX3 can perhaps swing the TGF- $\beta$ signaling pendulum from tumor promotion to tumor suppression.

\section{CONFLICTS OF INTEREST}

The authors declare no conflicts of interest.

Yoshiaki Ito: Cancer Science Institute of Singapore, National University of Singapore, Singapore-117599

Correspondence: Yoshiaki Ito, email csiitoy@nus.edu.sg

Keywords: TGF-beta, RUNX3, HMOX1, DNA damage

Received: November 21, 2017

Published: November 28, 2017

\section{REFERENCES}

1. Bierie B, et al. Nat Rev Cancer. 2006; 6:506-520.

2. Ito Y, et al. Nat Rev Cancer. 2015; 15:81-95.

3. Chi XZ, et al. Mol Cell Biol. 2005; 25:8097-8107.

4. Yano T, et al. Mol Cell Biol. 2006; 26:4474-4488.

5. Voon DC, et al. Stem Cells. 2012; 30:2088-2099.

6. Krishnan V, et al. Cancer Research. 2017 Oct 26. doi: 10.1158/0008-5472.CAN-17-1178. [Epub ahead of print].

7. Coppé JP, et al. Annu Rev Pathol. 2010; 5:99-118.

8. Wang CQ, et al. Cell Reports. 2014; 8:767-782.
Copyright: Krishnan et al. This is an open-access article distributed under the terms of the Creative Commons Attribution License 3.0 (CC BY 3.0), which permits unrestricted use, distribution, and reproduction in any medium, provided the original author and source are credited.

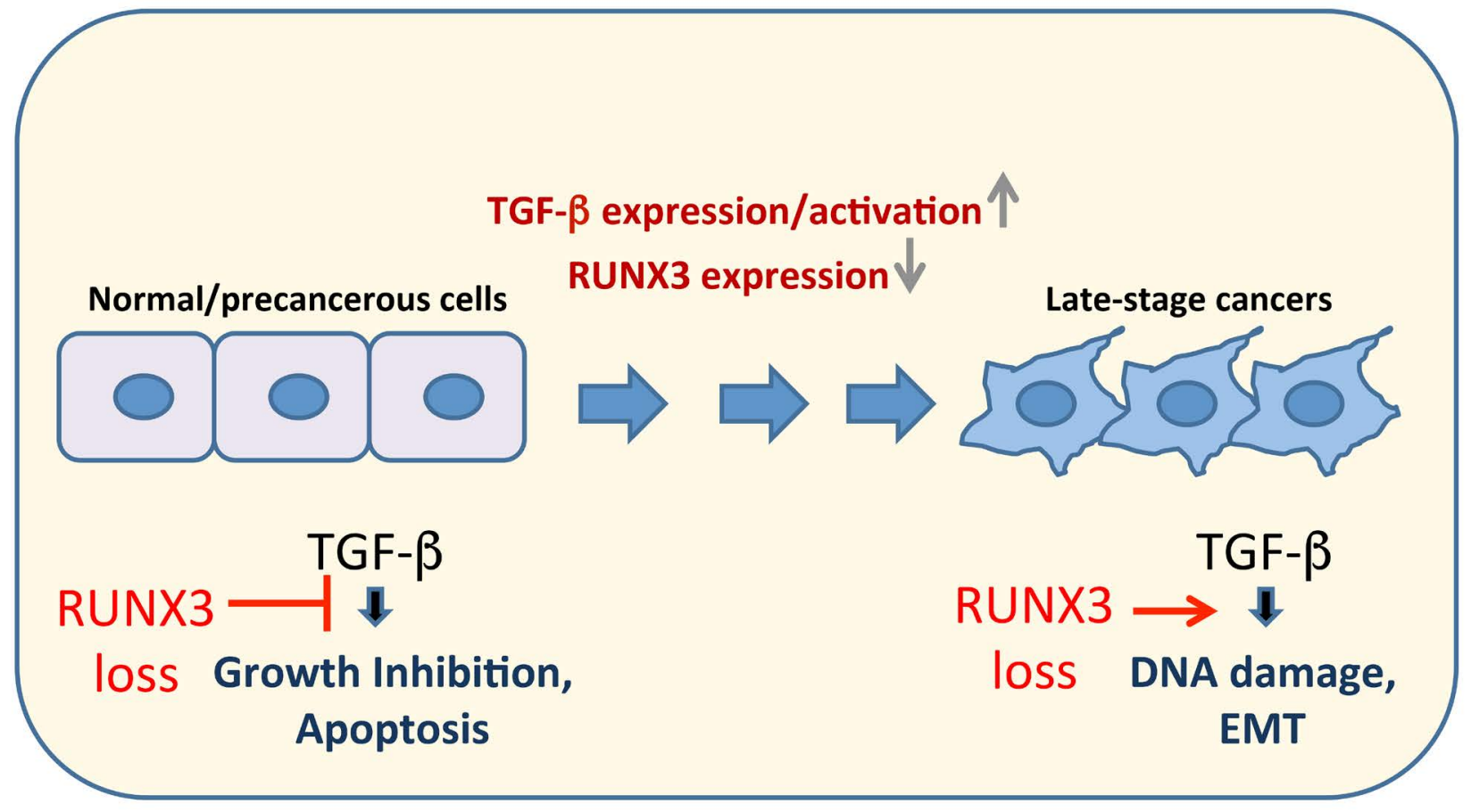

Figure 1: A model on how $R U N X 3$ loss promotes the pro-carcinogenic functions of TGF- $\beta$ signaling. 